\title{
Two-Dimensional Copolymers and Exact Conformal Multifractality
}

\author{
Bertrand Duplantier \\ Service de Physique Théorique de Saclay, F-91191 Gif-sur-Yvette Cedex \\ and Institut Henri Poincaré, 11, rue Pierre et Marie Curie, F-75231 Paris Cedex 05
}

(August 21, 1998)

\begin{abstract}
We consider in two dimensions (2D) the most general star-shaped copolymer, mixing random (RW) or selfavoiding walks (SAW) with specific mutual avoidance interactions thereof. Its exact conformal scaling dimensions in the plane are derived from an algebraic structure existing on a random lattice (2D quantum gravity). The multifractal dimensions $\tau(n)$ of the harmonic measure of a 2D RW or SAW are conformal dimensions of certain star copolymers. The exact associated $f(\alpha)$ are identical for a RW or a SAW in 2D. These are examples of conformal multifractality.

PACS numbers: 05.20.-y, 02.50.-r, 05.70.Jk, 64.60.Ak
\end{abstract}

The concepts of generalized dimensions and associated multifractal (Mf) measures have been developed more than a decade ago [1 4 . They are encountered in many physical situations: strange attractors in dynamical systems, growth phenomena, harmonic measure of diffusion-limited aggregates, electron localization, random resistors, and random spin systems. Recently, analytic progress has been made for turbulence of a passive scalar 5] or for a growth model of DLA [6], both where the Mf dimensions can be calculated perturbatively.

It is well known that universal geometrical fractals, e.g., random walks, polymers, Ising or percolation models are essentially related to standard critical phenomena and field theory, for which conformal invariance in two dimensions (2D) has brought a wealth of exact results. By contrast, few connections between multifractals and field theory have been found, although the algebras of their respective correlation functions reveal intriguing similarities [7]. It remains a challenge to see if an exact description of some multifractal phenomena could emerge in 2D from the conformal invariance classification.

A particularly interesting multifractal phenomenon was singled out some time ago by Cates and Witten [8]. They showed that the moments of the harmonic measure, i.e., the Laplacian diffusion field near an absorber, the latter taken as a simple random walk (RW, i.e., Brownian motion), or self-avoiding walk (SAW, i.e., polymer), exhibit in $d$ dimensions multifractal scaling for $d<4$. The associated exponents can be recast as those of star copolymers made of a bunch of independent RW's diffusing away from a generic point of the absorber. This allowed the perturbative calculation of the Mf spectrum through standard RG theory for polymers and the $\varepsilon=4-d$ expansion.
Star polymers or networks made of self-avoiding walks only and their scaling properties are well understood [9], especially in 2D, where all topology dependent exponents are known exactly from conformal invariance [9,10]. For simple random walks, scaling exponents describing RW non-intersections [11,12], conjectured in [13], have been recently derived using conformal invariance and the socalled quantum gravity method [14].

The aim of this Letter is to extend conformal invariance to arbitrary mixed copolymers, thereby providing the exact Mf harmonic spectrum of a RW or a SAW, an example of conformal multifractality.

Consider a general star copolymer $\mathcal{S}$ in the plane $\mathbb{R}^{2}$ (or in $\mathbb{Z}^{2}$ ), made of an arbitrary mixture of Brownian paths or RW's (set $\mathcal{B}$ ), and polymers or SAW's $($ set $\mathcal{P})$, all starting at neighboring points. Any pair $(A, B)$ of such paths, $A, B \in \mathcal{B}$ or $\mathcal{P}$, can be constrained in a specific way: either they avoid each other $(A \cap B=\emptyset$, noted $A \wedge B)$, or they are transparent and can cross each other (noted $A \vee B$ ), corresponding to four different fixed points [15]. This notation allows for any nested interaction structure; one can decide for instance that the branches $\left\{P_{\ell} \in \mathcal{P}\right\}_{\ell=1, \ldots, L}$ of an $L$-star polymer, all mutually avoiding, further avoid a bunch of Brownian paths $\left\{B_{k} \in \mathcal{B}\right\}_{k=1, \ldots, n}$, all transparent to each other:

$$
\mathcal{S}=\left(\bigwedge_{\ell=1}^{L} P_{\ell}\right) \wedge\left(\bigvee_{k=1}^{n} B_{k}\right)
$$

In $2 \mathrm{D}$ the order of the branches of the star copolymer does matter and is intrinsic to our $(\wedge, \vee)$ notation.

To each specific star copolymer center $\mathcal{S}$ is attached a conformal scaling operator with a scaling dimension $x(\mathcal{S})$. To obtain proper scaling we consider the Brownian paths and the polymers to have the same mean size $R$. It is convenient to define for each star $\mathcal{S}$ a grand canonical partition function [9, 11,16], with fugacities $z$ and $z^{\prime}$ for the total lengths $|\mathcal{B}|$ and $|\mathcal{P}|$ of Brownian or polymer-like paths:

$$
\mathcal{Z}_{R}(\mathcal{S})=\sum_{\mathcal{B}, \mathcal{P} \in \mathcal{S}} z^{|\mathcal{B}|} z^{|\mathcal{P}|} \mathbb{1}_{R}(\mathcal{S})
$$

where the set of walks of $\mathcal{S}$ is constrained by the indicatrix $\mathbb{I}_{R}(\mathcal{S})$ to stay within a disc of radius $R$ centered on the star. At the critical values $z_{c}=\mu_{B}^{-1}, z_{c}^{\prime}=$ $\mu_{P}^{-1}$, where for the RW's $\mu_{B}$ is the coordination number of the underlying lattice, and $\mu_{P}$ the effective one for the SAW's, $\mathcal{Z}_{R}$ decays as [9, 16] 


$$
\mathcal{Z}_{R}(\mathcal{S}) \sim R^{-x(\mathcal{S})-x^{\vee}}
$$

where $x(\mathcal{S})$ is associated only with the singularity occurring at the center of the star where all critical paths meet, while $x^{\vee}$ is the contribution of the independent dangling ends or split star. It reads $x^{\vee}=\|\mathcal{B}\| x_{B, 1}+\|\mathcal{P}\| x_{P, 1}-2 \mathcal{V}$, where $\|\mathcal{B}\|$ and $\|\mathcal{P}\|$ are respectively the total numbers of Brownian or polymer paths of the star; $x_{B, 1}$ or $x_{P, 1}$ are the scaling dimensions of the extremities of a single RW $x_{B, 1}=0$, or SAW $x_{P, 1}=\frac{5}{48}$ [9, 17]. The last term $\mathcal{V}=\|\mathcal{B}\|+\|\mathcal{P}\|$ corresponds in $(3)$ to the integration over extremity positions in the disc of radius $R$.

When the star is constrained to stay in a half-plane with its core placed near the boundary, its partition function scales as 9,10 .

$$
\tilde{\mathcal{Z}}_{R}(\mathcal{S}) \sim R^{-\tilde{x}(\mathcal{S})-x^{\vee}}
$$

where $\tilde{x}(\mathcal{S})$ is the boundary scaling dimension, $x^{\vee}$ staying the same for star extremities in the bulk.

Any scaling dimension $x$ in the bulk is twice the conformal dimension (c.d.) $\Delta^{(0)}$ of the corresponding operator, while near a boundary (b.c.d.) they are identical:

$$
x=2 \Delta^{(0)}, \quad \tilde{x}=\tilde{\Delta}^{(0)} .
$$

This Letter provides the main lines of a derivation of these exponents.

The idea is to use another representation where the RW's or SAW's are on a $2 \mathrm{D}$ random lattice, i.e., in the presence of 2D quantum gravity 18]. One can indeed put any 2D statistical system on a random planar graph, thereby obtaining a new critical behavior, corresponding to the confluence of the critical point of the infinite random graph with that of the original model. The partition function of the random graph $G$ made of, e.g., trivalent vertices, reads

$$
Z_{\chi}(\beta)=\sum_{G(\chi)} e^{-\beta|G|},
$$

where the sum extends over graphs $G$ with a given topology of Euler characteristic $\chi$, modulo the group of automorphisms of $G,|G|$ being the number of vertices. Near the critical point $\beta_{c}$ where $|G|$ becomes infinite

$$
Z_{\chi}(\beta) \sim\left(\beta-\beta_{c}\right)^{2-\gamma_{\mathrm{str}}(\chi)} ;
$$

$\gamma_{\mathrm{str}}(\chi)=2-\frac{5}{4} \chi$ is the string susceptibility exponent [19]. The partition function of the copolymer star $\mathcal{S}$ on the random lattice $G$, with the sphere topology $(\chi=2)$, is defined as:

$$
Z(\mathcal{S})=\sum_{G(\chi=2)} e^{-\beta|G|} \mathcal{Z}_{G}(\mathcal{S})
$$

where the partition function $\mathcal{Z}_{G}(\mathcal{S})$ is defined as in (2), with the indicatrix $\mathbb{1}_{R}$ for the star being confined to the disc of radius $R$ now being replaced by the indicatrix $\mathbb{1}_{G}$ of the star being embedded in $G$. The partition sum (8) is now three-fold grand canonical, depending implicitly on fugacities $e^{-\beta}, z$, and $z^{\prime}$. A further fugacity term has to be added when dealing with a random graph $G$ with the disc topology $(\chi=1)$ and boundary length $|\partial G|$, in order to define a boundary partition function

$$
\tilde{Z}(\mathcal{S})=\sum_{G(\chi=1)} e^{-\beta|G|-\beta^{\prime}|\partial G|} \tilde{\mathcal{Z}}_{G}(\mathcal{S}),
$$

where the core star is now on the boundary $\partial G$.

There exists a finite size scaling regime 20 where both the lattice and the walks become infinite, $\beta, \beta^{\prime}, z$, and $z^{\prime}$ approaching together their respective critical values in a well defined way. In this regime, the partition functions $Z, \tilde{Z}$, after normalization by the random surface ones (6), are expected to scale as [21]:

$$
\begin{aligned}
& Z(\mathcal{S}) / Z_{\chi=2}(\beta) \sim|G|^{-\Delta(\mathcal{S})-\Delta^{\vee}}, \\
& \tilde{Z}(\mathcal{S}) / Z_{\chi=1}(\beta) \sim|\partial G|^{-\tilde{\Delta}(\mathcal{S})}|G|^{-\Delta^{\vee}} .
\end{aligned}
$$

Here $|G| \sim\left(\beta-\beta_{c}\right)^{-1}$ is the average size of the random lattice, while $|\partial G| \sim|G|^{1 / 2}$ is the mean length of the boundary. $\Delta(\mathcal{S})$ and $\tilde{\Delta}(\mathcal{S})$ are respectively the bulk and boundary conformal dimensions of the star core, dressed by gravity. Finally the dimension $\Delta^{\vee}$ in (10,11) is associated with the star extremities, as was $x^{\sqrt{ }}$ in (33). Equations (10) and (11) are formally identical to (3) and (4) in the plane, after recalling (5) and identifying the star area $R^{2}$ in the plane to the random area $|G|$.

A general constitutive relation due to Knizhnik et al. exists between the conformal dimension $\Delta^{(0)}$ of a scaling operator in the plane and the c.d. $\Delta$ of the same operator on the random surface $\Delta^{(0)}=\Delta[1-(1-\Delta) / \kappa]$, where $\kappa$ is a parameter related to the central charge of the original statistical model in the plane $c=1-6(1-\kappa)^{2} / \kappa$ 18,22. For walks $c$ vanishes, whence $\kappa=3 / 2$, and

$$
\Delta^{(0)}=U(\Delta), \tilde{\Delta}^{(0)}=U(\tilde{\Delta}), U(x)=\frac{x}{3}(1+2 x),
$$

this relation holding also for boundary operators.

Here I give a set of basic underlying topological "surgery" rules which allow the mixing of geometrical operators on a random surface. The set of relations for walks is so stringent that it yields immediately the conformal dimensions of any copolymer star, both on a random surface and in the plane.

Star algebra: The bulk and boundary conformal dimensions, in the presence of gravity, satisfy:

$$
2 \Delta-\gamma_{\text {str }}(\chi=2)=\tilde{\Delta} .
$$

This fairly general relation can be derived from factorization properties of partition functions like (10,11) [14,21]. As a consequence, substituting relation (13) with $\gamma_{\text {str }}(\chi=2)=-\frac{1}{2}$ in (12) gives the planar bulk dimensions from the gravity boundary ones: 


$$
\Delta^{(0)}=U(\Delta)=V(\tilde{\Delta}), V(x)=\frac{1}{24}\left(4 x^{2}-1\right) .
$$

Consider now two sub-stars $A, B$, issued at the same point near a boundary line and avoiding each other. We state that their boundary dimensions $\tilde{\Delta}$ on the random surface are additive:

$$
\tilde{\Delta}(A \wedge B)=\tilde{\Delta}(A)+\tilde{\Delta}(B) .
$$

Owing to (13), a similar relation, but with a constant shift, exists for bulk exponents. On $G$, two elements in a pair $(A, B)$ of walk sets are indeed made non-intersecting by gluing a fluctuating patch of random surface between them. Each set, $A$ or $B$, defines its own independent disc as in (9), with additive b.c.d.'s (11,15). So, mutually avoiding sets are rendered independent by the fluctuations of a random surface. Thus relation (15) can be derived from factorization properties of exact partition functions [14,21.

Consider by contrast two sets $A, B$ of walks which are mutually transparent, i.e., $A \vee B$. In the half-plane $\mathbb{R}^{+} \times$ $\mathbb{R}$, they are independent, and their boundary dimensions obey a linear relation

$$
\tilde{\Delta}^{(0)}(A \vee B)=\tilde{\Delta}^{(0)}(A)+\tilde{\Delta}^{(0)}(B),
$$

due to the trivial factorization of their partition functions. On a random surface, their boundary dimensions are obtained by inverting (12)

$$
\tilde{\Delta}=U^{-1}\left(\tilde{\Delta}^{(0)}\right), U^{-1}(x)=\frac{1}{4}(\sqrt{24 x+1}-1),
$$

and are not additive. The metric fluctuations indeed couple the sets $A, B$.

It is clear at this stage that the set of equations above is complete. It allows for the calculation of any conformal dimensions $\Delta(\mathcal{S})$ or $\Delta^{(0)}(\mathcal{S})$ associated with a star structure $\mathcal{S}$ of the most general type, as in (11), involving $(\wedge, \vee)$ operations separated by nested parentheses. Any such structure can be systematically reduced: one starts from the outermost parenthesis set, and calculates b.c.d.'s of operations $(\wedge, \vee)$ by using (15) for $\wedge$ on the random surface $G$, and (16) for $\vee$ on the plane $\mathbb{R}^{2}$, while applying repeatedly the non-linear map $U: G \rightarrow \mathbb{R}^{2}$ (12), or its inverse $U^{-1}$ (17) to transfer to the proper space where the boundary dimension is a linear representation of $\wedge$ or $\vee$. At the end, one uses (14) to recover bulk dimensions.

Brownian-polymer exponents: The single extremity scaling dimensions are for a RW or a SAW near a Dirichlet boundary in $\mathbb{R}^{2}$ 9,23

$$
\tilde{\Delta}_{B}^{(0)}(1)=1, \tilde{\Delta}_{P}^{(0)}(1)=\frac{5}{8},
$$

or on $G$, using (17), $\tilde{\Delta}_{B}(1)=U^{-1}(1)=1, \tilde{\Delta}_{P}(1)=$ $U^{-1}\left(\frac{5}{8}\right)=\frac{3}{4}$. Because of the star algebra described above these are the only numerical seeds, i.e., generators, we need.

Stars can include bunches of $n$ copies of transparent RW's or $m$ transparent SAW's. Their b.c.d.'s in $\mathbb{R}^{2}$ are respectively, by using (16) and (18), $\tilde{\Delta}_{B}^{(0)}(n)=n$ and $\tilde{\Delta}_{P}^{(0)}(m)=\frac{5}{8} m$, from which the inverse mapping (17) to the random surface yields $\tilde{\Delta}_{B}(n)=U^{-1}(n)$ and $\tilde{\Delta}_{P}(m)=U^{-1}\left(\frac{5}{8} m\right)$. The star made of $L$ bunches $\ell \in\{1, \ldots, L\}$ of $n_{\ell}$ transparent RW's each, and $L^{\prime}$ bunches $\ell^{\prime} \in\left\{1, \ldots, L^{\prime}\right\}$ of $m_{\ell^{\prime}}$ SAW's, all mutually avoiding, has planar scaling dimensions owing to (12,14) and (15)

$$
\begin{aligned}
\tilde{\Delta}^{(0)}\left\{n_{\ell}, m_{\ell^{\prime}}\right\} & =U(\tilde{\Delta}), \Delta^{(0)}\left\{n_{\ell}, m_{\ell^{\prime}}\right\}=V(\tilde{\Delta}), \\
\tilde{\Delta}\left\{n_{\ell}, m_{\ell^{\prime}}\right\} & =\sum_{\ell=1}^{L} U^{-1}\left(n_{\ell}\right)+\sum_{\ell^{\prime}=1}^{L^{\prime}} U^{-1}\left(\frac{5}{8} m_{\ell^{\prime}}\right) .
\end{aligned}
$$

These exponents are invariant under permutation of the bunches of walks. The existence of such a relation has been found for RW's in [24], but with an unspecified $U$, which is here derived from quantum gravity and generalized to SAW's.

For a copolymer star $\mathcal{S}_{L, L^{\prime}}$ made of $L$ RW's and $L^{\prime}$ SAW's, all mutually avoiding $\left(\forall \ell, \ell^{\prime}, n_{\ell}=m_{\ell^{\prime}}=1\right)$, $\tilde{\Delta}\left(\mathcal{S}_{L, L^{\prime}}\right)=L+\frac{3}{4} L^{\prime}$ gives the c.d. in $\mathbb{R}^{2}$

$$
\begin{aligned}
& \tilde{\Delta}^{(0)}\left(\mathcal{S}_{L, L^{\prime}}\right)=\frac{1}{3}\left(L+\frac{3}{4} L^{\prime}\right)\left(1+2 L+\frac{3}{2} L^{\prime}\right) \\
& \Delta^{(0)}\left(\mathcal{S}_{L, L^{\prime}}\right)=\frac{1}{24}\left[4\left(L+\frac{3}{4} L^{\prime}\right)^{2}-1\right],
\end{aligned}
$$

recovering for $L=0$ the SAW exponents [10] and for $L^{\prime}=0$ the RW non-intersection exponents [14].

Disconnection exponents: Take any walk star $A$, with elementary boundary c.d. $\tilde{x}$ in $\mathbb{R}^{2}$. The star $\mathcal{S}_{n}=(\vee A)^{n}$ made of $n$ transparent copies of $A$ has b.c.d. $n \tilde{x}$ in $\mathbb{R}^{2}$, thus $U^{-1}(n \tilde{x})$ on $G$. Its bulk c.d. in $\mathbb{R}^{2}$ is, according to (5, 14), $x\left(\mathcal{S}_{n}\right)=2 V\left[U^{-1}(n \tilde{x})\right]$, which differs from $n x$, where $x=2 V\left[U^{-1}(\tilde{x})\right]$ is the bulk c.d. in $\mathbb{R}^{2}$. Their difference is the disconnection exponent, governing the conditioned probability $P_{R} \sim R^{-x\left(\mathcal{S}_{n}\right)+n x}$ that the union of $n$ copies does not disconnect the star origin from infinity, within a disc of radius $R$.

Multifractal harmonic measure: The harmonic measure $H(w)$ of a given set is the probability that a RW coming from infinity first hits the set (the absorber) at point $w$. When the set is a RW or a SAW of size $R$, the site average of its moments $H^{n}$ has been shown [8] to be represented by a copolymer star partition function of type (11) where the absorber avoids a bunch of $n$ independent RW's. More precisely $\sum_{w} H^{n}(w) \sim$ $\mathcal{Z}_{R}\left(\mathcal{S}_{\wedge} n\right) / \mathcal{Z}_{R}\left(\mathcal{S}_{\wedge} 1\right)$, where the absorber $\mathcal{S}$ is either the two-RW star $B \vee B$ or the two-SAW star $P \wedge P$, made of two non-intersecting SAW's. We have introduced the short-hand notation $\mathcal{S}_{\wedge} n \equiv \mathcal{S} \wedge(\vee B)^{n}$ describing the copolymer star made by the absorber $\mathcal{S}$ hit by the bunch $(\vee B)^{n}$ at the apex only. Owing to 
Eq.(3), we get the scaling $\sum_{w} H^{n}(w) \sim R^{-\tau(n)}$, where $\tau(n)=(n-1) D(n)=x\left(\mathcal{S}_{\wedge} n\right)-x\left(\mathcal{S}_{\wedge} 1\right)$ defines $($ annealed [6]) generalized dimensions $D(n)$. Our formalism 114,15, 17) immediately gives the scaling dimensions $x\left(\mathcal{S}_{\wedge} n\right)=2 V\left(\tilde{\Delta}(\mathcal{S})+U^{-1}(n)\right)$, where $\tilde{\Delta}(\mathcal{S})$ is as usual the quantum gravity boundary dimension of the absorber $\mathcal{S}$ alone. A simple calculation gives $\tau(n)$ and its Legendre transform $f(\alpha)+\tau(n)=\alpha n, \alpha=d \tau(n) / d n$

$$
\begin{aligned}
\tau(n) & =\frac{1}{2}(n-1)+y \frac{1}{24}(\sqrt{24 n+1}-5), \\
f(\alpha) & =\frac{1}{24}\left\{\frac{25}{2}+5 y-\frac{1}{2} y^{2}(2 \alpha-1)^{-1}-\alpha\right\},
\end{aligned}
$$

where $y \equiv 4 \tilde{\Delta}(\mathcal{S})-1$ is the only parameter encoding which absorber we consider (which can actually be any star tip). For a RW absorber, we have $\tilde{\Delta}(B \vee B)=$ $U^{-1}(2)=\frac{3}{2}$, while for a SAW $\tilde{\Delta}(P \wedge P)=2 \tilde{\Delta}_{P, 1}=$ $2 U^{-1}\left(\frac{5}{8}\right)=\frac{3}{2}$, thus $y=5$ in both cases. The coincidence of these two values tells us that in 2D the harmonic multifractal spectra $f(\alpha)$ of a random walk or a self-avoiding walk are identical. Their Mf spectra associated with walk ends [8] however differ, and are obtained using $y=3$ for a RW end, or $y=2$ for a SAW end.
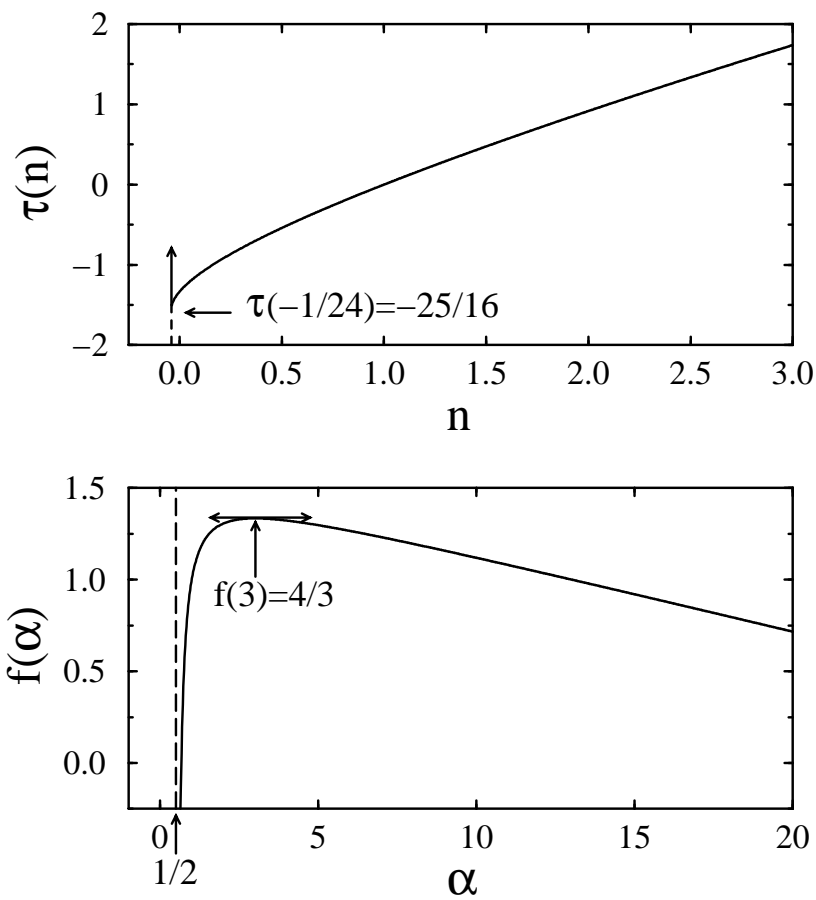

FIG. 1. Harmonic multifractal dimensions $\tau(n)$ and spectrum $f(\alpha)$ of a two-dimensional RW or SAW.

The corresponding universal curves for $y=5$ are shown in Fig.1: $\tau(n)$ is half a parabola, and $f(\alpha)$ a hyperbola. $D(1)=\tau^{\prime}(1)=1$ is just Makarov's theorem [25]; the divergence of $f$ at $\alpha_{\min }=\frac{1}{2}$ corresponds to singular needles in the absorber, while $-\tau(0)=\sup _{\alpha} f(\alpha)=f(3)=\frac{4}{3}$ is the Hausdorff dimension of the Brownian frontier or of a
SAW. Thus Mandelbrot's classical conjecture identifying the latter two is generalized and proven for the whole $f(\alpha)$ harmonic spectrum.

Acknowledgements. I thank T.C. Halsey for discussions and a careful reading of the manuscript.

[1] B.B Mandelbrot, J. Fluid. Mech. 62, 331 (1974).

[2] H.G.E. Hentschel and I. Procaccia, Physica (Amsterdam) 8D, 835 (1983).

[3] U. Frisch and G. Parisi, in Proceedings of the International School of Physics "Enrico Fermi", course LXXXVIII, edited by M. Ghil (North-Holland, New York, 1985) p. 84.

[4] T.C. Halsey, M.H. Jensen, L.P. Kadanoff, I. Procaccia, and B.I. Shraiman, Phys. Rev. A33, 1141 (1986).

[5] D. Bernard, K. Gawȩdzki, and A. Kupiainen, Phys. Rev. E54, 2564 (1996).

[6] T.C. Halsey, B. Duplantier, and K. Honda, Phys. Rev. Lett. 78, 1719 (1997); J. Stat. Phys. 85, 681 (1996).

[7] M. Cates and J.M. Deutsch, Phys. Rev. A35, 4907 (1987); B. Duplantier and A. Ludwig, Phys. Rev. Lett. 66, 247 (1991); C. von Ferber, Nucl. Phys. B490, 511 (1997).

[8] M.E. Cates and T.A. Witten, Phys. Rev. Lett. 56, 2497 (1986); Phys. Rev. A35, 1809 (1987).

[9] B. Duplantier, Phys. Rev. Lett. 57, 941 (1986); J. Stat. Phys. 54, 581 (1989).

[10] B. Duplantier and H. Saleur, Phys. Rev. Lett. 57, 3179 (1986); H. Saleur, J. Phys. A19 L807 (1986).

[11] B. Duplantier, Commun. Math. Phys. 117, 279 (1987).

[12] G.F. Lawler, Intersections of random walks (Boston, Birkhäuser, 1991).

[13] B. Duplantier and K.-H. Kwon, Phys. Rev. Lett. 61, 2514 (1988).

[14] B. Duplantier (Saclay preprint SPhT 98/063).

[15] J.F. Joanny, L. Leibler, and R.C. Ball, J. Chem. Phys. 81, 4640 (1984); L. Schäfer, U. Lehr, and C. Kappeler, J. Phys. I 1, 211 (1991).

[16] C. von Ferber and Y. Holovatch, Europhys. Lett. 39, 31 (1997); Phys. Rev. E56, 6370 (1997).

[17] B. Nienhuis, in Phase transitions and critical phenomena, Vol. 11, edited by C. Domb and J.L. Lebowitz (Academic, London, 1987).

[18] A.M. Polyakov, Mod. Phys. Lett. A2, 893 (1987); V.G. Knizhnik, A.M. Polyakov, and A.B. Zamolodchikov, Mod. Phys. Lett. A3, 819 (1988).

[19] I.K. Kostov and M.L. Mehta, Phys. Lett. B189, 118 (1987).

[20] B. Duplantier and I.K. Kostov, Phys. Rev. Lett. 61, 1436 (1988); Nucl. Phys. B340, 491 (1990).

[21] B. Duplantier (to be published).

[22] F. David, Mod. Phys. Lett. A3, 1651 (1988); J. Distler and H. Kawai, Nucl. Phys. B321 509 (1988).

[23] J. Cardy, Nucl. Phys. B240 [FS12], 514 (1984).

[24] G.F. Lawler and W. Werner (unpublished). 
[25] N.G. Makarov, Proc. London Math. Soc. 51, 369 (1985). 